•综述・

\title{
浅析环境特征对遗传多样性与 物种多样性的平行效应
}

\author{
徐武美 1,2 慈秀芹 1,2 李 $\quad$ 捷 $^{*}$ \\ 1 (中国科学院西双版纳热带植物园综合保护中心植物系统发育与保护生物学实验室, 昆明 650223) \\ 2 (中国科学院大学, 北京 100049)
}

\begin{abstract}
摘要: 遗传多样性与物种多样性是生物多样性的两个基本层次。近年来的研究表明, 环境特征可能对种群遗传多 样性与群落物种多样性产生平行效应。本文对遗传多样性与物种多样性关联模型中环境特征对遗传多样性与物种 多样性的平行效应部分进行了具体化介绍。基于群落形成的四个基本过程, 即突变与物种形成、选择、漂变和扩 散, 探讨了环境特征对遗传多样性与物种多样性产生平行效应的理论基础。在全球变化的大背景下, 研究环境特 征对遗传多样性与物种多样性的平行效应及这两个维度多样性对全球变化响应的异同具有重要的生态学与保护 生物学意义。然而, 目前大多数生物多样性研究仍然只基于物种多样性一个维度, 在同一系统中同时对遗传多样 性与物种多样性进行整合研究的工作仍然较少。希望通过本文的总结与讨论, 能对国内遗传多样性与物种多样性 整合研究起到参考与促进作用。
\end{abstract}

关键词：环境特征; 遗传多样性; 物种多样性; 平行效应; 全球变化; 生物多样性保护

\section{Parallel effects of environmental properties on genetic diversity and spe- cies diversity}

\author{
Wumei $\mathrm{Xu}^{1,2}$, Xiuqin $\mathrm{Ci}^{1,2}$, Jie $\mathrm{Li}^{1 *}$ \\ 1 Plant Phylogenetics and Conservation Group, Center for Integrative Conservation, Xishuangbanna Tropical Botanical \\ Garden, Chinese Academy of Sciences, Kunming 650223 \\ 2 University of Chinese Academy of Sciences, Beijing 100049
}

\begin{abstract}
Genetic diversity and species diversity are the two basic levels of biodiversity; recent studies have indicated the parallel effects of environment properties on these two levels of biodiversity. Here we introduce the species-genetic diversity correlation model regarding the parallel effects of environment properties on genetic diversity and species diversity. Based on the four basic processes in community ecology and population genetics (i.e. mutation and speciation, selection, drift, dispersal), we further discuss the theoretical foundations of the parallel effects of environment properties on these two levels of biodiversity. The studies conducted on these effects have shown significant importance in ecology and conservation biology, especially in the age of global change; however, few studies consider these two levels of biodiversity together but most consider only one in the biodiversity research so far. Therefore, we review the recent progress and outline the future directions in the study of the parallel effects of environmental properties on genetic diversity and species diversity. We hope this will benefit and promote the related studies in China.
\end{abstract}

Key words: environmental properties; genetic diversity; species diversity; parallel effects; global change; biodiversity conservation

遗传多样性与物种多样性是生物多样性的两 个基本层次, 前者主要体现在种群内个体间的遗传
变异程度(Ellegren \& Galtier, 2016), 后者主要体现 在一个群落内物种的数量以及每个物种的个体在

收稿日期: 2017-01-03; 接受日期: 2017-04-06

基金项目: 中国西南地区极小种群野生植物调查与种质保存(2017FY100100)课题二 “滇西南地区极小种群野生植物调查与种质采集”(2017FY100102)、 国家自然科学基金(31500454)及中科院研究所“一三五”项目(XTBG-T01)

*通讯作者 Author for correspondence. E-mail: jieli@xtbg.ac.cn 
群落中的均匀程度(Whittaker, 1972)。Antonovics (1976) 指出, 影响种群遗传多样性与群落物种多样 性的因素是相似的, 即种群遗传多样性与群落物种 多样性可能存在正相关性。然而, 由于遗传多样性 与物种多样性分属不同学科的研究内容, 所以关于 二者的关系研究在随后的二十多年里并没有实质 性的进展。而近十年来开展的一系列研究表明, 环 境特征可能对种群遗传多样性与群落物种多样性 产生平行效应(Vellend, 2003, 2004; Struebig et al, 2011; Wei \& Jiang, 2012; Lamy et al, 2013)。在全球变 化的大背景下, 研究环境特征对二者的平行效应具 有重要的生态学与保护生物学意义(Taberlet et al, 2012)。

Vellend和Geber (2005)系统总结了环境特征对 遗传多样性与物种多样性的平行效应, 并基于群落 形成的 4 个基本过程(突变与物种形成、漂变、扩散 和选择), 探讨了环境特征对遗传多样性与物种多 样性产生平行效应的理论基础(图1, 表1)。之后开展 的大量相关研究(如Vellend, 2010; 朱璧如和张大勇, 2011; Bertin et al, 2016; Fourtune et al, 2016; Vellend, 2016)或多或少地支持了Vellend和Geber (2005)的模 型, 同时也强有力地表明一些全球变化因素如气候 变化、生境破碎化、富营养化、人为干扰等不仅可 能对一定区域内的物种多样性产生显著影响, 同时 还可能会影响到群落内物种的遗传多样性, 而遗传 多样性的降低可能会影响到其适合度 (Reed \& Frankham, 2003), 从而进一步加剧物种局域灭绝的
风险(Moritz, 2002)。

本文拟通过对一些重要且广泛论及的环境因 素(如生境破碎化、富营养化等)对遗传多样性与物 种多样性的平行效应进行介绍, 希望能对国内相关 研究提供参考。为简明且易于比较, 本文中遗传多 样性采用基于分子标记(如SSR)的不同多态位点平 均等位基因数或种群内基因型丰富度(genotypic richness)表示(Peakall \& Smouse, 2012; Avolio \& Smith, 2013; Lamy et al, 2013; Xu et al, 2016a); 物 种多样性用物种丰富度表示, 即一个群落(或调查 样方)内物种的数量(Jarzyna \& Jetz, 2016)。

\section{1 生境面积与隔离程度的平行效应}

生境面积会对群落内物种的数量及种群大小 产生影响。随着生境面积的减少, 群落内不同种群 的规模也可能随之减小, 对于某些小种群而言, 很 可能由于遗传漂变而使种群内某些等位基因随机 丢失, 致使遗传多样性降低; 在遗传多样性降低的 同时, 也可能使一些个体数较小的种群产生局域灭 绝, 使群落物种多样性减少。然而, 由于等位基因 与物种以花粉、种子扩散等方式在种群及群落间的 移动, 又会使由于漂变而随机降低的种群遗传多样 性与群落物种多样性得以补充。因此, 生境面积与 隔离程度可能通过影响漂变、迁移等生态学过程, 对种群遗传多样性与群落物种多样性产生平行效 应(表1，图1)。根据岛屿生物地理学平衡理论的预测, 大的岛屿比小的岛屿能维持更多的物种数, 而随岛

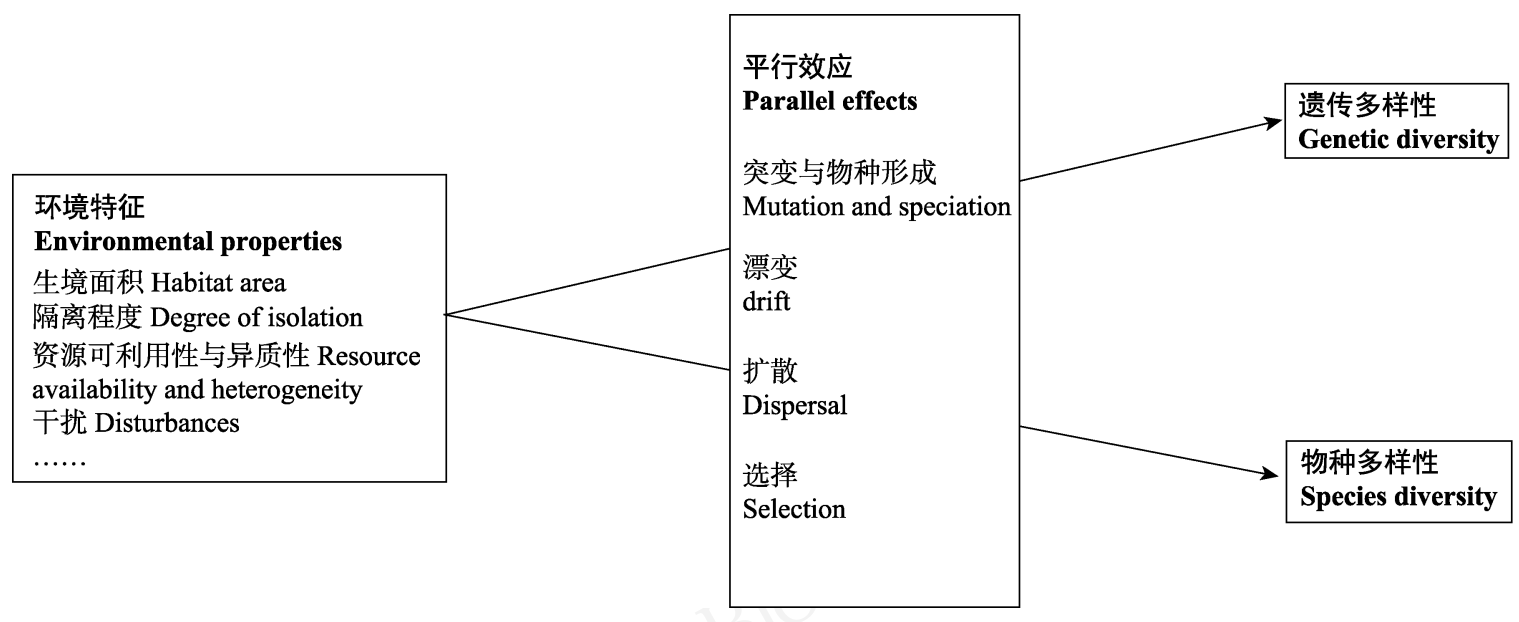

图1 环境特征对遗传多样性与物种多样性的平行效应理论模型(参考Vellend和Geber, 2005)

Fig. 1 A theoretical model which depict the parallel effects of environmental properties on genetic diversity and species diversity (refer to Vellend \& Geber, 2005) 
表1 不同生态过程对遗传多样性与物种多样性的平行效应(参考Vellend和Geber, 2005)

Table 1 The parallel effects of different ecological processes on genetic diversity and species diversity (refer to Vellend \& Geber, 2005)

\begin{tabular}{lll}
\hline 生态过程 Ecological processes & 遗传多样性 Genetic diversity & 物种多样性 Species diversity \\
\hline 突变与物种形成 Mutation and speciation & 新等位基因的产生 The creation of new alleles & 新物种的形成 The creation of new species \\
& 种群内等位基因频率的随机变化 & 群落内物种相对多度的随机变化 \\
& Random changes in the relative frequencies of & Random changes in the relative abundance of \\
& alleles within a population & species within a community \\
& 种群间等位基因的移动 & 群落间物种的移动 \\
& Movement of alleles among populations & Movement of species among communities \\
& 仅对种群内某些等位基因有利的生态过程 & 仅对群落内某些物种有利的生态过程 \\
& Processes that favour particular alleles over & Process that favour particular species over \\
& others within a population & others within a community \\
\hline
\end{tabular}
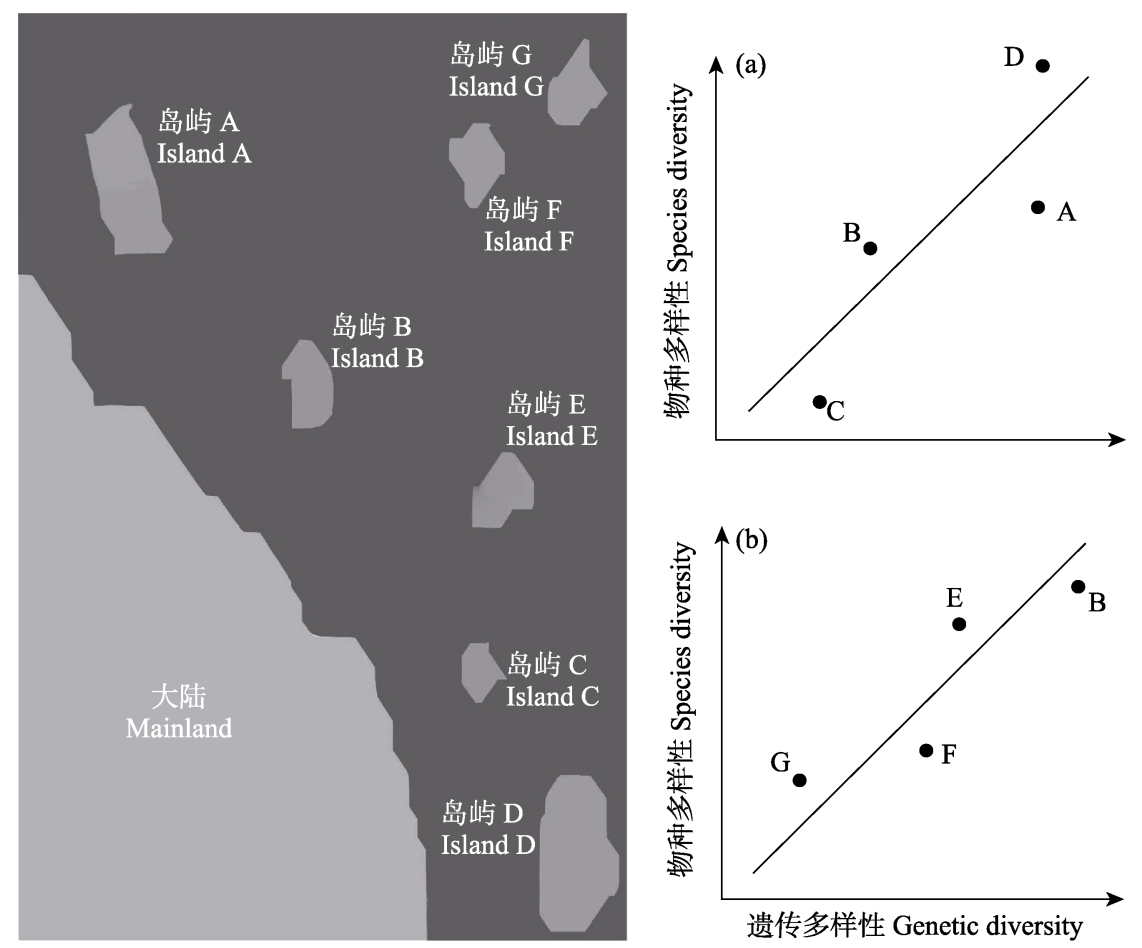

图2 根据平衡理论所预测的遗传多样性与物种多样性的正相关性(参考Vellend, 2003)。假设岛屿A、B、C、D与大陆的距离 相同, 而岛屿面积为 D $>$ A $>$ B $>$ C ; 岛屿B、E、F、G 具有相同的面积且距离大陆的距离(隔离程度)为 $\mathbf{G}>\mathbf{F}>\mathbf{E}>\mathbf{B}$ 。假定岛 屿之间不存在物种与基因流, 根据平衡理论预测, 生境面积(图2a)与隔离程度(图2b)是种群遗传多样性与群落物种多样性呈 正相关的驱动因素

Fig. 2 The predicted positive correlation between genetic diversity and species diversity according to the equilibrium theory (refer to Vellend, 2003). We assume that the island A, B, C, D have the same distance to the mainland while the area is D $>A>B>C$; the island B, E, F, G have the same area while the distances to the mainland are G $>$ F $>$ E $>$ B. We also assume that there is no gene and species flow among the islands. Based on the predictions of the equilibrium theory, habitat area (Fig. 2a) and degree of isolation (Fig. $2 b)$ are the drivers of the positive correlation between genetic diversity and species diversity.

屿离大陆的距离由近到远, 平衡点的物种数逐渐降 低(MacArthur \& Wilson, 1963; 戈峰, 2008)。同样, 因为大的岛屿比小的岛屿能维持更大的种群规模 并包含更多的遗传多样性, 而距离大陆更近的种群 可能由于新等位基因的加入而使遗传多样性水平 得以维持(图2)。
Vellend (2003)对14项基于岛屿的研究进行整 合分析, 发现各研究的目标种(focal species)种群遗 传多样性与群落物种多样性的相关系数中位数为 0.63 , 显著高于随机模型预测值, 为平衡理论所预 测的二者正相关性提供了直接证据。此外, 平衡理 论同样适用于预测生境破碎化对二者的平行效应 
(Fahrig, 2003)。Struebig等(2011)在马来西亚热带森 林的研究发现, 生境破碎化后斑块大小对群落物种 多样性及目标种Kerivoula papillosa种群遗传多样 性表现出平行效应; Lamy等(2013)通过对法国西部 地区淡水螺的研究发现, 生境连接度 (habitat connectivity)是目标种Drepanotrema depressissimum 和Aplexa marmorata遗传多样性与群落物种多样性 呈正相关性的主要驱动因素; Laroche等(2015)通过 数学模型对遗传多样性与物种多样性的关系进行 模拟, 发现当种群内个体突变速率较低时, 不同群 落间由于物种扩散能力存在差异而可能导致遗传 多样性与物种多样性呈正相关。

生境破碎化是导致全球生物多样性锐减的主 要因素之一(Fahrig, 2003)。理论与实例研究均表明, 生境破碎化会使种群遗传多样性与群落物种多样 性平行降低(Vellend \& Geber, 2005; Struebig et al, 2011; Leonardi et al, 2012; Haddad et al, 2015; Laroche et al, 2015)。因此, 在生境破碎化与生物多 样性的关联研究中, 有必要对遗传多样性与物种多 样性进行综合探讨, 以制定更加合理的生物多样性 保护策略。

\section{2 资源可利用性与异质性的平行效应}

群落内个体的生长与种群的维持都需要消耗 资源; 群落内不同的物种与种群内具有不同基因型 的个体, 在不同的生境中具有不同的选择优势 (Vellend \& Geber, 2005)。因此资源可利用性与异质 性可能对种群遗传多样性与群落物种多样性产生平 行效应(图1)。

\section{1 资源可利用性}

一般认为, 资源可利用性与物种多样性的关系 为单峰模式(Grime, 1973)。当资源可利用性很低时, 只有很少数的物种能够生存下来; 随着资源可利用 性增加, 群落内物种数增加到峰值; 而随着资源可 利用性进一步增加, 种间竞争也随之增加, 少数优 势种排斥其它共存种, 导致群落物种多样性降低。 资源可利用性与物种多样性的单峰模式关系, 对于 种群内具有不同基因型的个体也是适用的：当资源 极度匮乏时, 只有少数基因型能够存活; 随着资源 可利用性的不断增加, 少数基因型获得生长优势并 对其它基因型产生排斥。然而, 由于种间及种内存 在多种相互关系(Chu et al, 2008), 资源可利用性所
介导的遗传多样性与物种多样性的联系也显得更 加复杂(Silvertown et al, 2009; Xu et al, 2016a)。He 等(2008)对澳大利亚西部沙漠地区不同沙丘的物种 多样性及目标种Banksia attenuata的遗传多样性进 行了研究, 发现二者存在显著正相关, 进一步分析 揭示水分可利用性是其驱动因素。

\section{2 资源异质性}

一般认为, 群落内资源异质性(或环境异质性) 会促进物种多样性(Ricklefs, 1977; Stevens et al, 2012; Stein et al, 2014; Yang et al, 2015; 徐武美等, 2015); 同样, 资源异质性也是种群遗传多样性的重 要影响因素, 其机制与物种多样性相似(Gram \& Sork, 2001; Yeaman \& Jarvis, 2006; Huang et al, 2016)。Vellend和Geber (2005)基于Tilman (1982)资 源竞争模型, 讨论了资源异质性对遗传多样性与物 种多样性的平行效应。我们对Vellend和Geber (2005) 的模型进行了改进, 把资源可利用性代入模型。基 于改进后的模型可以看出, 资源异质性对种群遗传 多样性与群落物种多样性具有平行促进效应; 随着 群落内资源可利用性的不断增加, 最终导致它们一 同降低(图3)。

虽然理论预测资源(环境)异质性会对种群遗传 多样性与群落物种多样性产生平行促进作用, 然而 截至目前并没有直接证据表明二者会沿着一个异 质性梯度产生平行变化。这可能是由于以下两方面 的因素: (1)资源异质性与资源可利用性往往存在联 系, 致使它们对多样性的影响难以区分(Lundholm, 2009; Xu et al, 2016b); (2)种间相互作用可能会掩盖 资源异质性对种群遗传多样性与群落物种多样性 的平行效应，如随着群落内资源异质性增加导致更 多的物种得以共存, 然而更多物种的加入又可能导 致种间竞争增加, 使得平均种群规模减小, 遗传漂 变增强, 进而使遗传多样性降低(Vellend \& Geber, 2005; Xu et al, 2016a)。

当前, 人类活动正导致生物群落的资源可利用 性发生变化, 尤其是大气氮沉降与生境富营养化已 被证实是导致生物多样性锐减的重要因素(Wassen et al, 2005; Harpole \& Tilman, 2007; Clark \& Tilman, 2008; Hautier et al, 2009; Liu et al, 2013)。近年来已 有不少学者开始关注全球变化对遗传多样性的影 响(Pauls et al, 2013; Ravenscroft et al, 2015), 然而大 多数研究通常还只是针对物种多样性一个维度进 


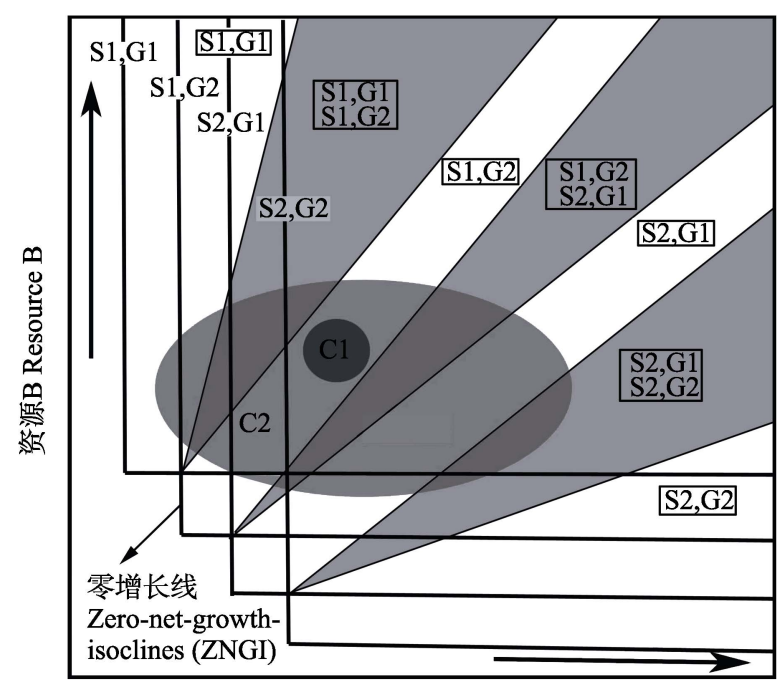

资源A Resource A

图3 基于Tilman资源竞争模型的资源可利用性与异质性对 种群遗传多样性与群落物种多样性的影响(参考Vellend和 Geber, 2005)。ZNGI为零增长线, 如果两个物种或同一物种 不同基因型的ZNGI线相交, 表明它们可能稳定共存, 但取 决于资源可利用性在二维空间的分布状况。方框内表示在不 同资源可利用性水平下可共存的物种或基因型。群落1(C1) 具有较低的环境异质性, 在这种环境下, 只有物种S1的一个 基因型G2 (S1, G2)存在竞争优势并长期存在; 群落2 (C2)具 有较高的环境异质性, 在这种环境下, 所有物种的所有基因 型都能够长期稳定共存。黑色箭头表示资源可利用性的增加 方向, 随着群落内资源可利用性的不断增加, 二维资源可利 用区域不断向上或向右移动, 最终由于资源可利用性增加 而导致种群遗传多样性与群落物种多样性一同降低。

Fig. 3 The predicted effects of resource availability and heterogeneity on genetic diversity within a population and species diversity within a community based on the Tilman (1982) model (refer to Vellend \& Geber, 2005). ZNGI indicates the zero-net-growth-isoclines, all points on the isocline, the reproductive rate of a species equals its mortality. The intersection point between the ZNGIs indicates the potential stable coexistence among the species with different genotypes which depend on the status of resource distribution within the community. The species/genotypes listed in boxes indicate those that will coexist at equilibrium given different possible resource supply points. In this model, community 1 (C1) with a low resource heterogeneity and only genotype 2 in species 1 (S1, G2) can survive and exclude the others; while in community two (C2), the high resource heterogeneity allow all the two genotypes (G1 and G2) in each of two species (S1 and S2) coexisted within the community. The black arrows indicate the directions for the increased resource availability, with the resource availability increased, both genetic diversity within population and species diversity within community decreased in parallel eventually.

行探讨(Jarzyna \& Jetz, 2016)。

\section{3 干扰的平行效应}

干扰对生物多样性的影响甚为广泛(Thom \&
Seidl, 2015; Yuan et al, 2016)。一般认为，中度干扰 有利于群落内物种共存, 而高强度的干扰则会使群 落物种多样性降低(Connell, 1978; Biswas \& Mallik, 2010)。同样, 中度干扰可能使种群内某些具有明显 竞争优势的基因型得到一定程度的抑制, 而使具有 不同基因型的个体得以共存; 随着干扰的频度与强 度增加, 只有少数具有明显抗干扰能力的基因型个 体得以存活(Vellend \& Geber, 2005)。因此预测, 干 扰可能对种群遗传多样性与群落物种多样性产生 平行效应(图1)。

关于干扰对种群遗传多样性与群落物种多样 性的平行效应, 已开展了较多的研究(Vellend, 2004; Cleary et al, 2006; Evanno et al, 2009; Wei \& Jiang, 2012; Frey et al, 2016), 主要分为人为干扰以及自然 干扰对这两个维度多样性的平行效应。在人为干扰 方面, Vellend (2004)对美国纽约中部地区原始森林 与次生林(森林砍伐作农业用地后废弃形成)进行了 调查, 发现干扰后的次生林草本植物群落物种多样 性及目标种Trillium grandiflorum的遗传多样性均较 原始林更低, 并提出土地利用历史(人为干扰与否) 是遗传多样性与物种多样性正相关的驱动因素; Frey等(2016)调查了法国大西洋沿岸沙丘生态系统 7 种常见植物的遗传多样性与群落物种多样性, 发 现人为干扰(娱乐设施修建等)是导致其遗传多样性 与物种多样性平行降低的主要原因。在自然干扰方 面, Cleary等(2006)对印度尼西亚东Kalimantan地区 蝴蝶群落进行了研究, 发现厄尔尼诺现象所介导的 自然干扰是蝴蝶群落物种多样性及目标种Drupadia theda遗传多样性呈正相关的驱动因素; Evanno等 (2009)在法国东南部地区的研究发现, 自然干扰(极 度干旱) 是导致该地区腹足类动物物种多样性及目 标种Radix balthica遗传多样性平行下降的驱动因 素。然而, 干扰也不总是对遗传多样性与物种多样 性产生平行效应, 比如Wei和Jiang (2012)研究了中 国神农架地区沿海拔梯度树种多样性及优势种领 春木(Euptelea pleiospermum)遗传多样性的相关性, 发现海拔是二者正相关的驱动因素，同时发现人为 干扰主要影响了群落树种多样性而非领春木种群 遗传多样性, 导致在干扰生境中二者的相关性不 显著。

干扰对种群遗传多样性与群落物种多样性的 影响是两方面的: 中度干扰使多样性上升, 而高强 
度干扰导致多样性降低(Connell, 1978)。截至目前, 虽然大部分研究仅关注干扰对种群遗传多样性与 群落物种多样性的协同负作用, 而近年来却有研究 表明, 干扰是物种多样性得以维持的关键因素 (Yuan et al, 2016)。因此，探讨干扰是不是同时促进 了这些区域物种的遗传多样性, 将有助于进一步理 解中度干扰对遗传多样性与物种多样性的多方面 影响。

\section{4 遗传多样性与物种多样性的纬度分布格局}

纬度作为多种生态因子的复合体, 对生物多样 性分布格局产生间接影响(Gaston, 2000)。从全球尺 度看, 物种多样性呈纬度梯度分布格局(latitudinal biodiversity gradient, LBG), 即与高纬度地区相比, 低纬度地区(如热带雨林)具有更高的物种多样性 (Gaston, 2000; Willig et al, 2003; Dowle et al, 2013) (图4)。对于物种多样性的纬度梯度分布格局有超过 25种解释(Gaston, 2000), 然而并没有一致性的结论 (Dowle et al, 2013)。一般认为, 由于低纬度地区具 有更好的水热条件、更稳定的环境特征从而具有更 高的成种速率(rates of speciation)和较低的灭绝速率 (Rohde, 1992; Willig et al, 2003; Dowle et al, 2013); 同时，低纬度地区具有更大的地球表面积及更高的 环境异质性(Ricklefs, 1977), 因而有助于多物种的 稳定共存。

与物种多样性的纬度梯度分布格局相似, 遗传 多样性也呈现出类似的纬度梯度分布格局, 具体表 现为低纬度地区物种遗传多样性较高而高纬度地 区物种遗传多样性较低(Eo et al, 2008; Vik et al, 2010; Inza et al, 2012; Adams \& Hadly, 2013; Araujo \& Costa-Pereira, 2013)。遗传多样性与物种多样性在 纬度梯度分布上的耦合暗示着可能存在一些共同 的驱动因素(Schrey et al, 2011; Pope et al, 2015), 导 致遗传多样性与物种多样性在纬度梯度上协同变 化。Vellend和Geber (2005) 提出突变与物种形成是遗 传多样性与物种多样性平行变化的驱动因素之一 (表1); 然而, 由于突变与物种形成发生在不同的时 间尺度，因此研究者没有深入探讨其对二者的平行 效应。遗传多样性与物种多样性的纬度梯度分布格 局可能为此提供了间接的证据(Pope et al, 2015)。根 据进化速率假设 (evolutionary speed hypothesis) (Rohde, 1992; Dowle et al, 2013), 与高纬度地区相

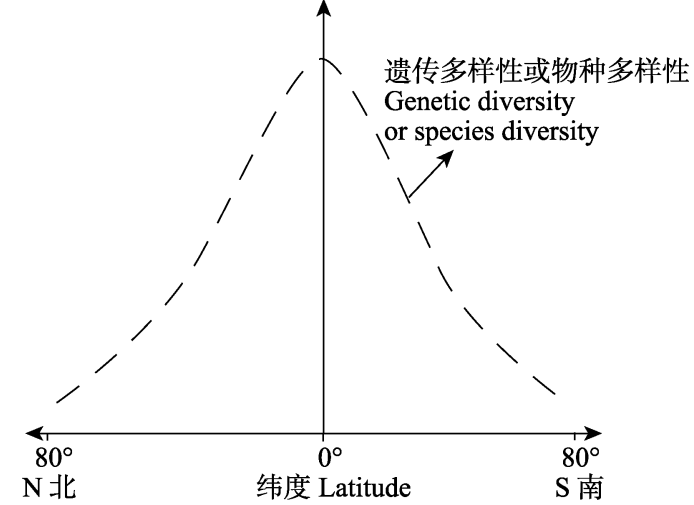

图4 根据进化速率假设所预测的遗传多样性与物种多样性 的纬度梯度分布格局 (参考Rohde，1992; Gaston， 2000; Araujo \& Costa-Pereira, 2013; Dowle et al, 2013)

Fig. 4 The latitudinal patterns of genetic diversity and species diversity based on the predictions of evolutionary speed hypothesis (refer to Rohde, 1992; Gaston, 2000; Araujo \& Costa-Pereira, 2013; Dowle et al, 2013)

比, 低纬度地区(如热带雨林)物种具有更高的突变 (进化)速率，而高的突变速率一方面加速了种群内 新基因型的产生，同时也加速了新物种的形成(图4)。

\section{5 展望}

如上所述, 环境特征确有可能对种群遗传多样 性与群落物种多样性产生平行效应。在全球变化的 大背景下，我们不仅需要关注全球性的环境问题 (如生境破碎化、富营养化等)对物种多样性的影响, 同时也迫切需要了解其它维度的生物多样性(如遗 传多样性)对这些环境问题的响应。因此，基于多维 度生物多样性的整合研究, 就显得尤为迫切(张全 国等, 2014)。Vellend和Geber (2005)系统总结了环境 特征对遗传多样性与物种多样性的平行效应, 在国 际上引起了很大的反响; 国内在这一领域的研究虽 然取得了一些进展(Wei \& Jiang 2012; Xu et al, 2016a), 但仍亟待加强。尤其是随着中国经济不断 发展, 由此带来的环境压力也会不断增加, 其对生 物多样性的影响需要多方面衡量(Bellard et al, 2012)。例如, Liu等(2013)研究了中国1980-2010年间 大气氮沉降的基本情况, 发现全国范围内大气氮沉 降越来越严重, 而氮沉降的增加又显著改变了植物 氮可利用性水平。根据Tilman的资源竞争理论(图3), 氮可利用性的增加不仅会影响到群落内物种多样 性，还会对物种的遗传多样性产生影响(Vellend \& Geber, 2005), 如果只关注大气氮沉降与物种多样 
性的联系(Clark \& Tilman, 2008), 就很可能忽视其 对遗传多样性的影响。希望通过本文的总结与讨论, 能引起国内相关学者对遗传多样性与物种多样性 整合研究的关注, 基于不同学科的交叉与融合, 系 统探讨环境变化对生物多样性的多方面影响, 从而 制定更加合理的保护策略。

致谢: 感谢Richard T Corlett研究员在中国科学院西 双版纳热带植物园2017年第二届高级科学论文写 作研修班期间对本文英文摘要进行的修改。

\section{参考文献}

Adams RI, Hadly EA (2013) Genetic diversity within vertebrate species is greater at lower latitudes. Evolutionary Ecology, 27, 133-143.

Antonovics J (1976) The input from population genetics: "the new ecological genetics”. Systematic Botany, 1, 233-245.

Araujo MS, Costa-Pereira R (2013) Latitudinal gradients in intraspecific ecological diversity. Biology Letters, 9, 20130778.

Avolio ML, Smith MD (2013) Correlations between genetic and species diversity: effects of resource quantity and heterogeneity. Journal of Vegetation Science, 24, 1185-1194.

Bellard C, Bertelsmeier C, Leadley P, Thuiller W, Courchamp F (2012) Impacts of climate change on the future of biodiversity. Ecology Letters, 15, 365-377.

Bertin A, Gouin N, Baumel A, Gianoli E, Serratosa J, Osorio R, Manel S (2016) Genetic variation of loci potentially under selection confounds species-genetic diversity correlations in a fragmented habitat. Molecular Ecology, 26, 431-443.

Biswas SR, Mallik AU (2010) Disturbance effects on species diversity and functional diversity in riparian and upland plant communities. Ecology, 91, 28-35.

Chu CJ, Maestre FT, Xiao S, Weiner J, Wang YS, Duan ZH, Wang G (2008) Balance between facilitation and resource competition determines biomass-density relationships in plant populations. Ecology Letters, 11, 1189-1197.

Clark CM, Tilman D (2008) Loss of plant species after chronic low-level nitrogen deposition to prairie grasslands. Nature, 451, 712-715.

Cleary DF, Fauvelot C, Genner MJ, Menken SB, Mooers AO (2006) Parallel responses of species and genetic diversity to El Nino Southern Oscillation-induced environmental destruction. Ecology Letters, 9, 304-310.

Connell JH (1978) Diversity in tropical rain forests and coral reefs-high diversity of trees and corals is maintained only in a non-equilibrium state. Science, 199, 1302-1310.

Dowle EJ, Morgan-Richards M, Trewick SA (2013) Molecular evolution and the latitudinal biodiversity gradient. Heredity, 110, 501-510.

Ellegren H, Galtier N (2016) Determinants of genetic diversity. Nature Reviews Genetics, 17, 422-433.

Eo SH, Wares JP, Carroll JP (2008) Population divergence in plant species reflects latitudinal biodiversity gradients. Biology Letters, 4, 382-384.

Evanno G, Castella E, Antoine C, Paillat G, Goudet J (2009) Parallel changes in genetic diversity and species diversity following a natural disturbance. Molecular Ecology, 18, 1137-1144.

Fahrig L (2003) Effects of habitat fragmentation on biodiversity. Annual Review of Ecology, Evolution, and Systematics, 34, 487-515.

Fourtune L, Paz-Vinas I, Loot G, Prunier JG, Blanchet S (2016) Lessons from the fish: a multi-species analysis reveals common processes underlying similar species-genetic diversity correlations. Freshwater Biology, 61, 1830-1845.

Frey D, Arrigo N, Granereau G, Sarr A, Felber F, Kozlowski G (2016) Parallel declines in species and genetic diversity driven by anthropogenic disturbance: a multispecies approach in a French Atlantic dune system. Evolutionary Applications, 9, 479-488.

Gaston KJ (2000) Global patterns in biodiversity. Nature, 405, 220-227.

Ge F (2008) Modern Ecology. Science Press, Beijing. (in Chinese) [戈峰 (主编) (2008) 现代生态学. 科学出版社, 北京. ]

Gram WK, Sork VL (2001) Association between environmental and genetic heterogeneity in forest tree populations. Ecology, 82, 2012-2021.

Grime JP (1973) Competitive exclusion in herbaceous vegetation. Nature, 242, 344-347.

Haddad NM, Brudvig LA, Clobert J, Davies KF, Gonzalez A, Holt RD, Lovejoy TE, Sexton JO, Austin MP, Collins CD, Cook WM, Damschen EI, Ewers RM, Foster BL, Jenkins CN, King AJ, Laurance WF, Levey DJ, Margules CR, Melbourne BA, Nicholls AO, Orrock JL, Song DX, Townshend JR (2015) Habitat fragmentation and its lasting impact on Earth's ecosystems. Science Advances, 1, e1500052.

Harpole WS, Tilman D (2007) Grassland species loss resulting from reduced niche dimension. Nature, 446, 791-793.

Hautier Y, Niklaus PA, Hector A (2009) Competition for light causes plant biodiversity loss after eutrophication. Science, 324, 636-638.

He T, Lamont BB, Krauss SL, Enright NJ, Miller BP (2008) Covariation between intraspecific genetic diversity and species diversity within a plant functional group. Journal of Ecology, 96, 956-961.

Huang Y, Tran I, Agrawal AF (2016) Does genetic variation maintained by environmental heterogeneity facilitate adaptation to novel selection? The American Naturalist, 188, 27-37.

Inza MV, Zelener N, Fornes L, Gallo LA (2012) Effect of lati- 
tudinal gradient and impact of logging on genetic diversity of Cedrela lilloi along the Argentine Yungas Rainforest. Ecology and Evolution, 2, 2722-2736.

Jarzyna MA, Jetz W (2016) Detecting the multiple facets of biodiversity. Trends in Ecology and Evolution, 31, 527-538.

Lamy T, Jarne P, Laroche F, Pointier JP, Huth G, Segard A, David P (2013) Variation in habitat connectivity generates positive correlations between species and genetic diversity in a metacommunity. Molecular Ecology, 22, 4445-4456.

Laroche F, Jarne P, Lamy T, David P, Massol F (2015) A neutral theory for interpreting correlations between species and genetic diversity in communities. The American Naturalist, 185, 59-69.

Leonardi S, Piovani P, Scalfi M, Piotti A, Giannini R, Menozzi P (2012) Effect of habitat fragmentation on the genetic diversity and structure of peripheral populations of beech in central Italy. Journal of Heredity, 103, 408-417.

Liu XJ, Zhang Y, Han WX, Tang AH, Shen JL, Cui ZL, Vitousek P, Erisman JW, Goulding K, Christie P, Fangmeier A, Zhang FS (2013) Enhanced nitrogen deposition over China. Nature, 494, 459-462.

Lundholm JT (2009) Plant species diversity and environmental heterogeneity: spatial scale and competing hypotheses. Journal of Vegetation Science, 20, 377-391.

MacArthur RH, Wilson EO (1963) An equilibrium theory of insular zoogeography. Evolution, 17, 373-387.

Moritz C (2002) Strategies to protect biological diversity and the evolutionary processes that sustain it. Systematic Biology, 51, 238-254.

Pauls SU, Nowak C, Balint M, Pfenninger M (2013) The impact of global climate change on genetic diversity within populations and species. Molecular Ecology, 22, 925-946.

Peakall R, Smouse PE (2012) GenAlEx 65: genetic analysis in Excel-Population genetic software for teaching and research—an update. Bioinformatics, 28, 2537-2539.

Pope LC, Riginos C, Ovenden J, Keyse J, Blomberg SP (2015) Population genetic diversity in the Australian "seascape": a bioregion approach. PLoS ONE, 10, e0136275.

Ravenscroft CH, Whitlock R, Fridley JD (2015) Rapid genetic divergence in response to 15 years of simulated climate change. Global Change Biology, 21, 4165-4176.

Reed DH, Frankham R (2003) Correlation between fitness and genetic diversity. Conservation Biology, 17, 230-237.

Ricklefs RE (1977) Environmental heterogeneity and plant species diversity: a hypothesis. The American Naturalist, 111, 376-381.

Rohde K (1992) Latitudinal gradients in species diversity: the search for the primary cause. Oikos, 65, 514-527.

Schrey AW, Grispo M, Awad M, Cook M, McCoy ED, Mushinsky H, Albayrak T, Bensch S, Burke T, Butler LK, Dor R, Fokidis HB, Jensen H, Imboma T, Kessler-Rios MM, Marzal A, Stewart IRK, Westerdahl H, Westneat DF, Zehtindjiev P, Martin LB (2011) Broad-scale latitudinal pat- terns of genetic diversity among native European and introduced house sparrow (Passer domesticus) populations. Molecular Ecology, 20, 1133-1143.

Silvertown J, Biss PM, Freeland J (2009) Community genetics: resource addition has opposing effects on genetic and species diversity in a 150-year experiment. Ecology Letters, 12, 165-170.

Stein A, Gerstner K, Kreft H (2014) Environmental heterogeneity as a universal driver of species richness across taxa, biomes and spatial scales. Ecology Letters, 17, 866-880.

Stevens RD, Gavilanez MM, Tello JS, Ray DA (2012) Phylogenetic structure illuminates the mechanistic role of environmental heterogeneity in community organization. Journal of Animal Ecology, 81, 455-462.

Struebig MJ, Kingston T, Petit EJ, Le Comber SC, Zubaid A, Mohd-Adnan A, Rossiter SJ (2011) Parallel declines in species and genetic diversity in tropical forest fragments. Ecology Letters, 14, 582-590.

Taberlet P, Zimmermann NE, Englisch T, Tribsch A, Holderegger $\mathrm{R}$, Alvarez $\mathrm{N}$, Niklfeld $\mathrm{H}$, Coldea $\mathrm{G}$, Mirek $\mathrm{Z}$, Moilanen A, Ahlmer W, Marsan PA, Bona E, Bovio M, Choler P, Cieslak E, Colli L, Cristea V, Dalmas JP, Frajman B, Garraud L, Gaudeul M, Gielly L, Gutermann W, Jogan N, Kagalo AA, Korbecka G, Kupfer P, Lequette B, Letz DR, Manel S, Mansion G, Marhold K, Martini F, Negrini R, Nino F, Paun O, Pellecchia M, Perico G, Piekos-Mirkowa H, Prosser F, Puscas M, Ronikier M, Scheuerer M, Schneeweiss GM, Schonswetter P, Schratt-Ehrendorfer L, Schupfer F, Selvaggi A, Steinmann K, Thiel-Egenter C, van Loo M, Winkler M, Wohlgemuth T, Wraber T, Gugerli F, IntraBioDiv Consortium, Vellend M (2012) Genetic diversity in widespread species is not congruent with species richness in alpine plant communities. Ecology Letters, 15, 1439-1448.

Thom D, Seidl R (2015) Natural disturbance impacts on ecosystem services and biodiversity in temperate and boreal forests. Biological Reviews, 91, 760-781.

Tilman D (1982) Resource Competition and Community Structure. Princeton University Press, Princeton.

Vellend M (2003) Island biogeography of genes and species. The American Naturalist, 162, 358-365.

Vellend M (2004) Parallel effects of land-use history on species diversity and genetic diversity of forest herbs. Ecology, 85, 3043-3055.

Vellend M (2010) Conceptual synthesis in community ecology. Quarterly Review of Biology, 85, 183-206.

Vellend M (2016) The Theory of Ecological Communities. Princeton University Press, Princeton.

Vellend M, Geber MA (2005) Connections between species diversity and genetic diversity. Ecology Letters, 8, 767-781.

Vik U, Jorgensen MH, Kauserud H, Nordal I, Brysting AK (2010) Microsatellite markers show decreasing diversity but unchanged level of clonality in Dryas octopetala (Rosaceae) with increasing latitude. American Journal of Botany, 97, 
988-997.

Wassen MJ, Venterink HO, Lapshina ED, Tanneberger F (2005) Endangered plants persist under phosphorus limitation. Nature, 437, 547-550.

Wei X, Jiang M (2012) Contrasting relationships between species diversity and genetic diversity in natural and disturbed forest tree communities. New Phytologist, 193, 779-786.

Whittaker RH (1972) Evolution and measurement of species diversity. Taxon, 21, 213-251.

Willig MR, Kaufman DM, Stevens RD (2003) Latitudinal gradients of biodiversity: pattern, process, scale, and synthesis. Annual Review of Ecology, Evolution, and Systematics, 34, 273-309.

Xu WM, Song CY, Li QM (2015) Relationship between soil resource heterogeneity and tree diversity in Xishuangbanna tropical seasonal rainforest, Southwest China. Acta Ecologica Sinica, 35, 7756-7762. (in Chinese with English abstract) [徐武美, 宋彩云, 李巧明 (2015) 西双版纳热带 季节雨林土壤养分空间异质性对乔木树种多样性的影响. 生态学报, 35, 7756-7762.]

Xu WM, Liu L, He T, Cao M, Sha LQ, Hu YH, Li QM, Li J (2016a) Soil properties drive a negative correlation between species diversity and genetic diversity in a tropical seasonal rainforest. Scientific Reports, 6, 20652.

Xu WM, Ci XQ, Song CY, He TH, Zhang WF, Li QM, Li J (2016b) Soil phosphorus heterogeneity promotes tree species diversity and phylogenetic clustering in a tropical sea- sonal rainforest. Ecology and Evolution, 6, 8719-8726.

Yang ZY, Liu XQ, Zhou MH, Ai D, Wang G, Wang YS, Chu CJ, Lundholm JT (2015) The effect of environmental heterogeneity on species richness depends on community position along the environmental gradient. Scientific Reports, 5, 15723.

Yeaman S, Jarvis A (2006) Regional heterogeneity and gene flow maintain variance in a quantitative trait within populations of lodgepole pine. Proceedings of the Royal Society B: Biological Sciences, 273, 1587-1593.

Yuan ZY, Jiao F, Li YH, Kallenbach RL (2016) Anthropogenic disturbances are key to maintaining the biodiversity of grasslands. Scientific Reports, 6, 22132.

Zhang QG, Zhang DY, Ge JP, Zhao GL, Yu ZL (2014) Progress and hotspot in integrative biodiversity research A review for "Shuangqing Forum, No. 88". Chinese Bulletin of Life Sciences, 26, 97-99. (in Chinese) [张全国, 张大勇, 葛剑平, 赵桂玲, 于振良 (2014) 生物多样性整合研究的 进展及热点——第 88 期 “双清论坛” 综述. 生命科学, 26 , 97-99.]

Zhu BR, Zhang DY (2011) A process-based theoretical framework for community ecology. Biodiversity Science, 19, 389-399. (in Chinese with English abstract) [朱璧如, 张大勇 (2011) 基于过程的群落生态学理论框架. 生物 多样性, 19, 389-399.]

(责任编委: 陈小勇 责任编辑: 时意专) 